J.Pharm.Dyn., 6, 888-891 (1983)

\title{
TRANSPORT CHARACTERISTICS OF $p$-ACETAMIDOBENZOIC ACID IN BRUSH BORDER MEMBRANE OF RAT SMALL INTESTINE
}

\author{
Masato Yasuhara, ${ }^{*}$ TOSHIKIRO KimURA ${ }^{* *}$ AND Hitoshi SeZAKI \\ Faculty of Pharmaceutical Sciences, Kyoto University, Yoshida Shimoadachi-cho, Sakyo-ku, Kyoto, 606, \\ Japan
}

(Received July 16, 1983)

Brush border membrane vesicles of rat small intestinal mucosa were prepared by the modified calcium precipitation method in order to investigate the intestinal secretion mechanism of $p$-acetamidobenzoic acid (Ac-PABA). The release rate of $\left[{ }^{14} \mathrm{C}\right]-\mathrm{Ac}-\mathrm{PABA}$ from preloaded vesicles was much faster than that of $p$-aminobenzoic acid (PABA) and was enhanced by the addition of unlabelled Ac-PABA in the outer medium. In contrast, the membrane transfer rate of Ac-PABA across the egg lecithin liposomes was slower than that of PABA. The contribution of a carrier-mediated transport mechanism in the brush border membrane to the intestinal secretion of Ac-PABA was suggested.

Keywords - intestinal secretion; membrane transport; brush border membrane; counter transport effect; liposome; $p$-acetamidobenzoic acid; $p$-aminobenzoic acid; rat

Our previous in vitro and in situstudies on the intestinal absorption of $p$-aminobenzoic acid (PABA) demonstrated that PABA is rapidly absorbed from the intestinal lumen and is subject to the metabolism by $N$-acetyltransferase in the intestinal epithelial cells. ${ }^{1-3)} p$-Acetamidobenzoic acid (Ac-PABA), the metabolite of PABA, was found to be excreted in the intestinal lumen predominantly. The transfer rate of AcPABA from the intracellular compartment to the luminal side calculated by an analog computer was much faster than that of the opposite direction. ${ }^{2)}$ Such a marked difference in the permeability of Ac-PABA through the brush border membrane based on the transfer direction could not be explained by a simple lipoid-barrier model. The efficient excretion of a metabolite formed in the epithelial cell across the brush border membrane seems to be rational for the presystemic elimination of a foreign compound.

The present studies were designed to compare the transport characteristics of Ac-PABA through the brush border membrane with those of $\mathrm{PABA}$ in order to elucidate the excretion mechanism of Ac-PABA in rat small intestine.

\section{MATERIALS AND METHODS}

Materials - $p$-Aminobenzoic acid and $p$ acetamidobenzoic acid were obtained from Nakarai Chemicals Co. (Japan). $\left[{ }^{14} \mathrm{C}\right]-p$-Aminobenzoic acid $(38 \mathrm{mCi} / \mathrm{mmol})$ and $\left[{ }^{14} \mathrm{C}\right]$ - $p$-acetamidobenzoic acid $(6.7 \mathrm{mCi} / \mathrm{mmol})$ were generous gifts of Dr. Toshio Nanbo, Daiichi Pure Chemicals Co., Japan. All other chemicals were of the finest grade available.

Preparation of Brush Border Membrane Fractions - Brush border membrane fraction of rat small intestinal mucosa was prepared by the $\mathrm{Ca}^{2+}$ precipitation procedure modified by Kessler et al.4) The purity of the obtained brush border membrane fraction was checked by the determination of alkaline phosphatase activity ${ }^{5)}$ and $\mathrm{Na}^{+}-\mathrm{K}^{+}$-ATPase activity ${ }^{6)}$ The protein concentration was determined by the method of

* Present address: Department of Pharmacy, Kyoto University Hospital, Sakyo-ku, Kyoto, 606, Japan.

** Present address: Faculty of Pharmaceutical Sciences, Okayama University, Tsushima naka 1-1-1, Okayama, 700, Japan. 
Lowry et al. with bovine serum albumin as the standard. ${ }^{\text {) }}$

Transport Measurements - The uptake of substrates by brush border membrane vesicles was determined by the rapid filtration technique as described by Hopfer et al. ${ }^{8)}$ Incubations were performed at $25^{\circ} \mathrm{C}$ in a buffer containing 100 mM mannitol, $10 \mathrm{mM} N$-2-hydroxyethylpiperazine- $N^{\prime}$-2-ethanesulfonic acid (HEPES)/ Tris at $\mathrm{pH} 7.5$ (buffer A). At selected times, incubation mixtures $(20 \mu \mathrm{l}$, about $100 \mu \mathrm{g}$ membrane protein) were diluted in $2 \mathrm{ml}$ of ice-cold stop solution (150 mM NaCl-1 mM HEPES/Tris, $\mathrm{pH}$ 7.5). The diluted sample was immediately transferred onto a glass filter (GC-50, $0.5 \mu \mathrm{m}$ pore size, Toyo Roshi Co., Japan) and rapidly rinsed with $10 \mathrm{ml}$ ice-cold stop solution. In order to determine the release rate of a drug from the membrane vesicles, brush border membrane vesicles were preincubated in buffer $A$ containing ${ }^{14} \mathrm{C}$-labelled substance for $60 \mathrm{~min}$ at $25^{\circ} \mathrm{C}$. Then, $100 \mu \mathrm{l}$ of preloaded membranes were diluted into $750 \mu$ l of buffer A without any drugs and incubated at $25^{\circ} \mathrm{C}$. Aliquots of $50 \mu \mathrm{l}$ were taken at selected times and the amount of a drug remained in vesicles was determined similarly as in the uptake experiments. In the case of short time release experiments, preloaded membrane vesicles $(10 \mu \mathrm{l})$ were diluted into $100 \mu \mathrm{l}$ of buffer $\mathrm{A}$ and incubated at $25^{\circ} \mathrm{C}$. Then, $2 \mathrm{ml}$ of ice-cold stop solution was added and immediately transferred onto a glass filter. The radioactivity of dried filters was determined by a liquid scintillation counting.

Preparation of Liposomes and Transfer Rate Measurement — The lipid thin layer film consisted of egg phosphatidylcholine (80), cholesterol (20) and dicetylphosphate $(5 \mu \mathrm{mol})$ was mixed with $5 \mathrm{ml}$ of drug solution (drug concentration, $40 \mathrm{mM}$ ) and the liposomal suspension was prepared by the method previously described. The overall transfer rate of drugs from liposomal suspensions was determined by the dynamic dialysis method. ${ }^{9)}$ Drug concentrations were determined spectrophotometrically as previously described. ${ }^{1)}$

\section{RESULTS AND DISCUSSION}

The purity of brush border membranes isolated from rat small intestinal mucosa was followed by measuring the activity of the marker enzymes. The specific activity of alkaline phosphatase, a typical brush border enzyme, was $9.2 \mathrm{U} / \mathrm{mg}$ of protein. When compared with the activities present in the original mucosal homogenates, this represented a 9.3-fold purification. In contrast, the specific activity of $\mathrm{Na}^{+}-\mathrm{K}^{+}$-ATPase, a marker enzyme of basolateral membrane, was enriched by a factor of 0.62 . Using these vesicles, the mode of D-glucose transport across the brush border membrane was investigated. The simultaneous addition of D-glucose $(1 \mathrm{mM})$ and $\mathrm{NaCl}$ (120 mM) to brush border membrane vesicles produced a transient 2.2-fold accumulation above the equilibrium level by the $\mathrm{Na}^{+}$-gradient induced increase in the initial uptake rate of Dglucose (overshoot phenomenon). This result of D-glucose transport by brush border membrane vesicles was in agreement with the data obtained by Hopfer et al. ${ }^{8)}$ Therefore, prepared vesicles proved to be suited for transport studies.

When membrane vesicles preloaded with labelled PABA (0.1 mM) or Ac-PABA (0.8 mM) were diluted into a medium without any drugs, the release of Ac-PABA was much faster than that of PABA (Fig. 1). At $1 \mathrm{~min}$ after the dilution, about $50 \%$ of preloaded Ac-PABA was released from the brush border membrane vesicles. In contrast, more than $50 \%$ of preloaded PABA remained in the vesicles at $30 \mathrm{~min}$. The question of whether the preloaded drugs located in an intravesicular space or in binding with membrane components is important. Therefore, the uptake at $60 \mathrm{~min}$ was measured when the intravesicular space was decreased by increasing the medium osmolarity with cellobiose. The uptake of AcPABA was proportional to the inverse osmolarity and, thus, to the intravesicular space. Consequently, the measured release of Ac-PABA could be accounted for completely by transport from the intravesicular space to the outer medium. On the other hand, the preloaded PABA would have existed in binding with mem- 
branes and have a slow release rate, since the uptake of PABA was nearly independent to the medium osmolarity change. Even after correcting the membrane binding effect by subtracting the amount in the vesicles at $30 \mathrm{~min}$ from those data at earlier period, the release rate of AcPABA from the brush border membrane vesicles was significantly faster than that of PABA.

In order to characterize the specificity of AcPABA transport in brush border membrane, the permeability of drugs through the simple lipid bilayer was measured using lecithin liposome dispersions as model membrane (Fig. 2). The transfer rate of Ac-PABA across the liposomal

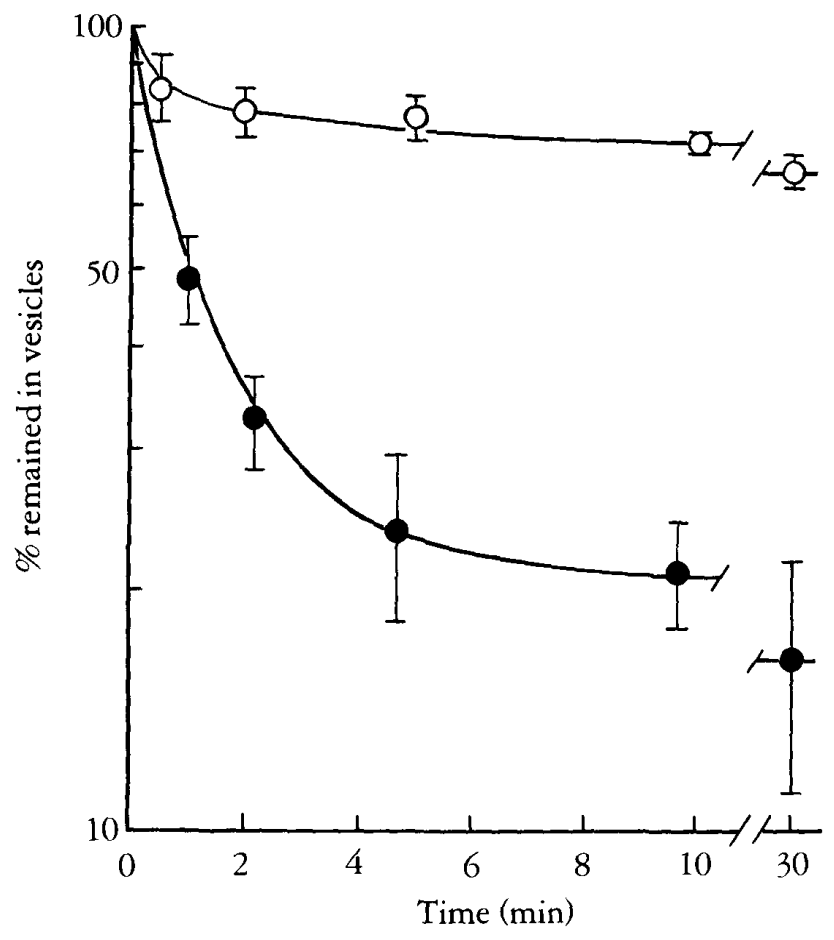

FIG. 1. Release of Drugs from Preloaded Brush Border Membrane Vesicles

The membrane was preloaded with $0.8 \mathrm{mM}$ $\left[{ }^{14} C\right]-A c-P A B A$ or $0.1 \mathrm{mM}\left[{ }^{14} C\right]-P A B A$ by incubation at this drug concentration for $I \mathrm{~h}$ at $25^{\circ} \mathrm{C}$. At zero time an aliquot of the preloaded membrane was diluted 8.5-fold into a medium containing no drugs. Drugs remaining in the membrane were measured by the rapid filtration technique, and the amount was calculated relative to an undiluted control at zero time.

- $A C-P A B A, \bigcirc: P A B A$. membrane was slower than that of PABA (release rate constant: Ac-PABA, $2.89 \times 10^{-2}$; PABA, $4.79 \times 10^{-2} \mathrm{~min}^{-1}$ ). Hence, the rapid release of Ac-PABA from the brush border membranes could not be explained by the simple diffusion mechanism.

Furthermore, when membrane vesicles preloaded with ${ }^{14} \mathrm{C}$-Ac-PABA $(0.1 \mathrm{mM})$ were diluted into a medium containing unlabelled AcPABA (1 $\mathrm{mM})$, the enhanced release of AcPABA was observed, indicating the counter transport effect (Fig. 3). At the initial phase (10 s), the release of labelled Ac-PABA was stimulated 2-fold by the addition of unlabelled AcPABA in the outer medium. These results sug-

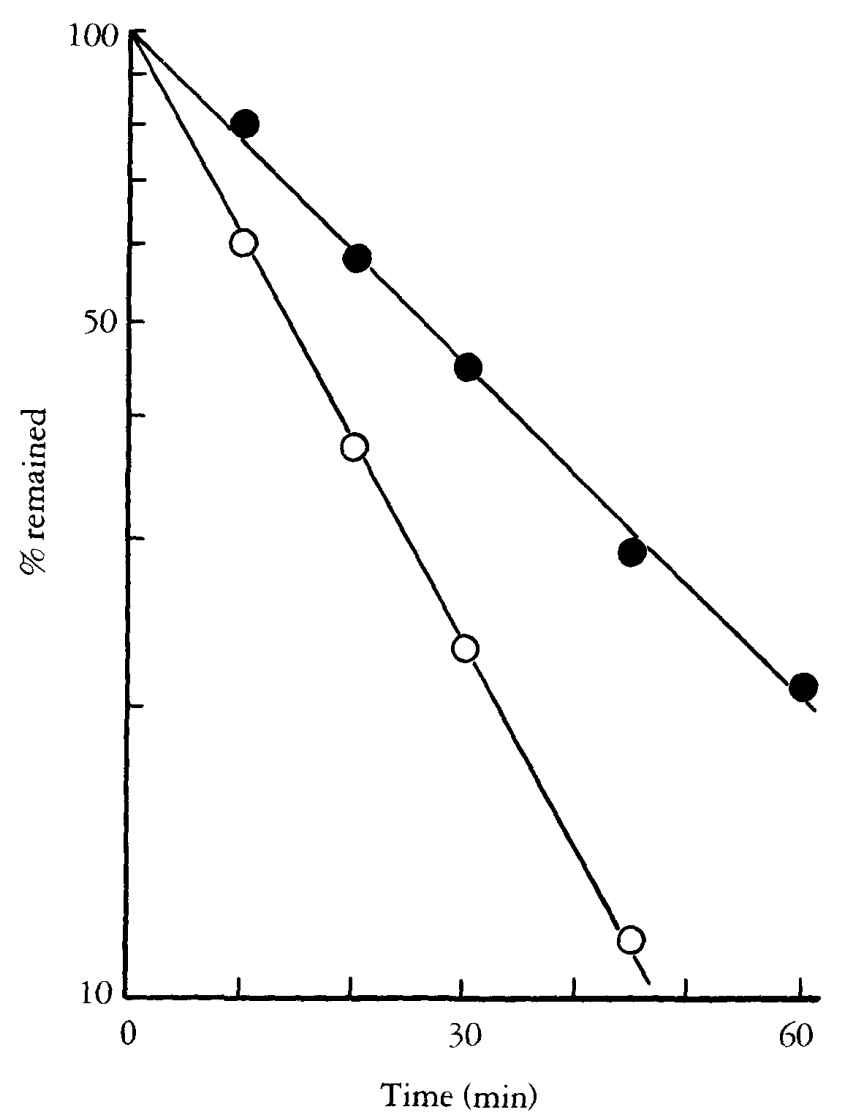

FIG. 2. Overall Transfer Rate of Drugs from Aqueous Liposome Dispersions Prepared from Egg Lecithin

Release of drugs from liposome was measured by the dynamic dialysis method at $\mathrm{pH} 6.5,37^{\circ} \mathrm{C}$.

- : Ac-PABA, O :PABA. 


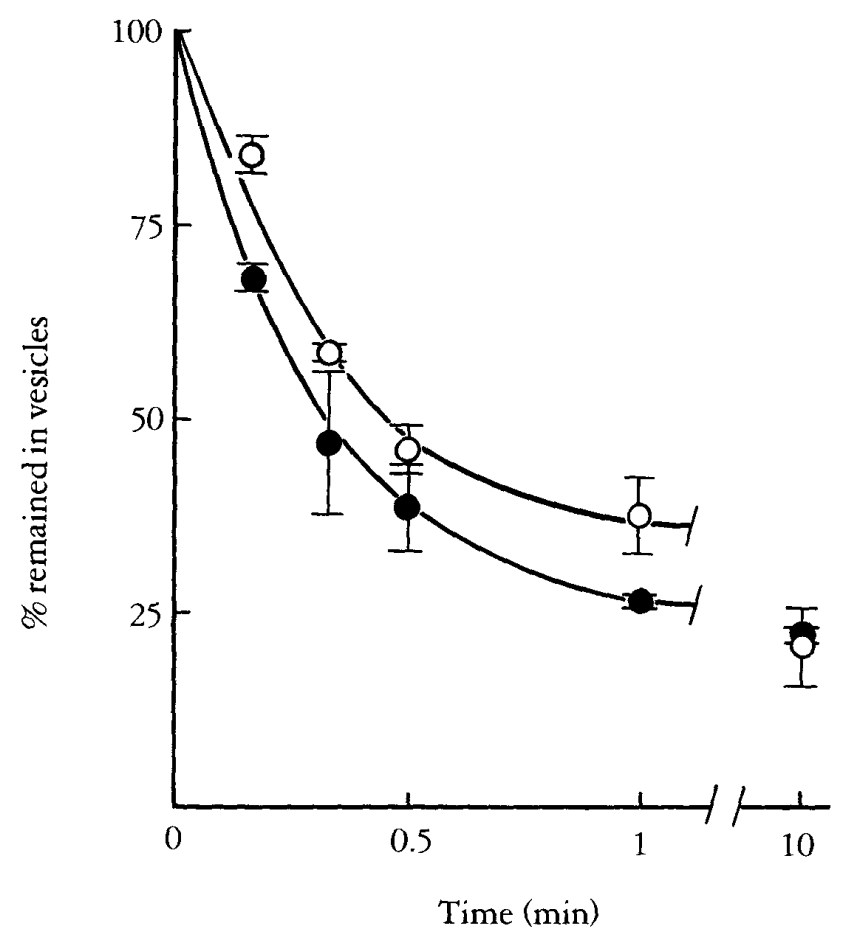

FIG. 3. Counter Transport Effect on Release of AcPABA from Preloaded Brush Border Membrane Vesicles

The membrane preloaded with $0.1 \mathrm{mM}\left[{ }^{14} \mathrm{C}\right]$ Ac-PABA was diluted 11-fold into a medium which contained no drug (control) or $1 \mathrm{mM}$ unlabelled Ac-PABA.

$\bigcirc$ : control, $\bigcirc$ : addition of $1 \mathrm{mM} \mathrm{Ac-PABA}$ in outer medium.

gest that Ac-PABA can be released into the outer medium by a carrier-mediated transport system in brush border membranes. The membrane vesicles prepared by the modified calcium pricipitation method was demonstrated that essentially all vesicles are right side out by Kessler et al. ${ }^{4)}$ Therefore, the observed release of Ac$\mathrm{PABA}$ can be considered reflecting the secretion of Ac-PABA from intracellular space into the intestinal lumen across the brush border membrane.

In addition, the transfer rate of Ac-PABA from serosal side to mucosal side in the intestinal sac was found to be saturable with increasing concentration of Ac-PABA (data not shown), which also suggests the presence of a carriermediated secretion system for Ac-PABA in the intestine.

Thus, Ac-PABA formed in the intestinal epithelial cells during the absorption process of PABA can be efficiently secreted into the luminal side by the carrier-mediated transport in the brush border membrane.

\section{REFERENCES}

1) M.Yasuhara, H.Kobayashi, S.Muranishi, H.Sezaki and T.Kimura: Absorption and metabolism of $p$-aminobenzoic acid by rat intestine in vitro, J. Pharm. Dyn., 1, 114-121 (1978).

2) M.Yasuhara, H.Kobayashi, T.Kimura, S.Muranishi and H.Sezaki: Absorption and metabolism of $p$-aminobenzoic acid by rat small intestine in situ, J. Pharm. Dyn., 1, 122-131 (1978).

3) M.Yasuhara, H.Kobayashi, Y.Kurosaki, T.Kimura, S.Muranishi and H.Sezaki: Comparative studies on the absorption mechanism of $p$-aminobenzoic acid and $p$-acetamidobenzoic acid from the rat intestine, $J$. Pharm. Dyn., 2, 177-186 (1979).

4) M.Kessler, O.Acuto, C.Storelli, H.Murer, M.Müller and G.Semenza: A modified procedure for the rapid preparation of efficiently transporting vesicles from small intestinal brush border membranes, Biochim. Biophys. Acta, 506, 136-154 (1978).

5) M.M.Weiser: Intestinal epithelial cell surface membrane glycoprotein synthesis, J. Biol. Chem., 248, 2536-2541 (1973).

6) T.Nakano, N.Nagano, K.Adachi and M.Nakao: Separation of two adenosine triphosphatases from erythrocyte membrane, Biochim. Biophys. Res. Commun., 13, 444-448 (1963).

7) O.H.Lowry, N.J.Resebrough, A.L.Farr and R.J.Randall: Protein measurement with the Folin phenol reagent, $J$. Biol. Chem., 193, 265-275 (1951).

8) U.Hopfer, K.Nelson, J.Perrotto and K.J.Isselbacher: Glucose transport in isolated brush border membrane from rat small intestine, J. Biol. Chem., 248, 25-32 (1973).

9) T.Kimura, M.Yoshikawa, M.Yasuhara and H.Sezaki: The use of liposomes as a model for drug absorption: $\beta$ lactam antibiotics, J. Pharm. Pharmacol., 32, $394-398(1980)$. 